\title{
Restoration and Enhancement of Underwater Image based on Wavelength Compensation and Image Dehazing Technique
}

\author{
Rubi Mandal, Prof.Sitendra Tamrakar \\ ${ }^{1}$ Mtech IVth SEM, CSE, NIIST Bhopal, India \\ ${ }^{2}$ Dept.CSE, NIIST Bhopal, India
}

\begin{abstract}
Getting clear images in underwater environments is an important issue in ocean engineering. The quality of underwater images plays a important role in scientific world. Capturing images underwater is difficult, generally due to deflection and reflection of water particles, and color change due to light travelling in water with different wavelengths. Light dispersion and color transform result in contrast loss and color deviation in images acquired underwater. Restoration and Enhancement of an underwater object from an image distorted by moving water waves is a very challenging task. This paper proposes wavelength compensation and image dehazing technique to balance the color change and light scattering respectively. It also removes artificial light by using depth map technique. Water depth is estimated by background color. Color change compensation is done by residual energy ratio method. A new approach is presented in this paper. We make use of a special technique called wavelength compensation and dehazing technique along with the artificial light removal technique simultaneously to analyze the raw image sequences and recover the true object. We test our approach on both pretended and data of real world, separately. Such technique has wide applications to areas such.
\end{abstract}

Keywords: Underwater image, color change, light scattering, depth map, wavelength compensation, image dehazing, image enhancement and restoration.

\section{Introduction}

Light dispersion and color change are two major sources of distortion for underwater image. Light scattering is caused by light incident on objects reflected and deflected multiple times by particles present in the water . This in turn lowers the visibility and contrast of the image captured. Color change occurs due to light traveling in the water with different wavelengths causing underwater area conquered by a bluish tone. None of the techniques can handle light spreading and color transform distortions suffered by underwater images, and the presence of non-natural lighting simultaneously. This paper suggests a organized approach to improve underwater images by a dehazing algorithm, to compensate the inconsistency along the propagation path by wavelength compensation technique, and to take the influence of the possible occurrence of an artifical light source into consideration. Once the distances between the objects and the camera is calculated, the front and background within a scene are segmented. The light intensities of front and back scenes are compared to determine whether an artificial light source is used. After neutralizing the effect of non-natural light, the haze phenomenon and change in wavelength attenuation along the underwater propagation path are rectified. Next, the water deepness in the image scene is found according to the residual energy ratios of different color channels existing in the background light. Based on the light wavelength, color change compensation is done to repair color. The performance of the proposed algorithm for wavelength compensation and image dehazing (WCID) is evaluated both objectively and subjectively by utilizing ground-truth color values.Underwater light is affected by deepness, subject distance, atmospheric and surface condition. Over the last few years, many researchers have generated various image enhancement and restoration algorithms for enhancing the quality of images captured in underwater environments. The three general phases that all types of data have to undergo while using digital technique are former processing, enhancement and display, information extraction.Conventionally,the processing of underwater images focuses solely on compensating either light scattering or color change distortion. An organized approach is needed to take all the factors concerning scattering of light, colour change, and probable presence of synthetic light source into consideration. Artificial light is removed by using depth map.

\section{Depth Map}

Dark-channel prior ,a scene-depth derivation method, is used first to calculate the distances of the scene objects to the camera. First an image is kept in an array. Next it is compared to the image of the other camera by taking square regions of pixels and comparing the intensity between the two cameras images. Third the depth of a given pixel region is calculated and kept in an array. Finally this array of depths is changed into color for 
maximum clarity, with bright colors being closer and dull colors being more far. Complete and well lit objects are the best to find the distance. Large blocks of solid color will produce black or nearly indiscriminate results. Calculate Depth

Now the depth value (between 0 and 255) is converted to a color to better show how far away the object is. Red is the closest in color list to black as the furthest. The real depths these colors symbolize is dependent upon your cameras and their distance from each other. The algorithm for wavelength compensation and image dehazing (WCID) proposed in this paper combines techniques of WCID to remove distortions caused by light scattering and color change.

\section{Light Scattering and Color Change}

Haze is caused by suspended particles such as sand, minerals and plankton that exist in lakes, oceans, and rivers. As light got reflected from objects propagates toward the camera, a portion of the light meets these suspended particles. This in turn absorbs and scatters the light beam, as illustrated in Fig. 1.Techniques targeting on removal of light scattering distortion include I. Exploiting the polarization effects to compensate for visibility degradation ii. Using image dehazing to restore the clarity of the underwater images and iii. Combining point spread functions and a modulation transfer function to reduce the blurring effect. Although the above mentioned approaches can enhance scene contrast and increase visibility, distortion caused by the disparity in wavelength attenuation, i.e. color change, remains intact. On the other hand, color-change correction techniques estimate underwater environmental parameters by performing color registration with consideration of light attenuation i. Employing histogram equalization in both RGB and HSI color spaces to balance the luminance distributions of color. ii. Wavelenght Compensation Method and iii. dynamically mixing the illumination of an object in a distance-dependent way by using a controllable multicolor light source to compensate color loss. Despite the improved color balance, these methods are ineffective in removing the image blurriness caused by light scattering. A systematic approach is needed to take all the factors concerning light dispersion, color modification, and possible occurrence of synthetic light source into consideration.

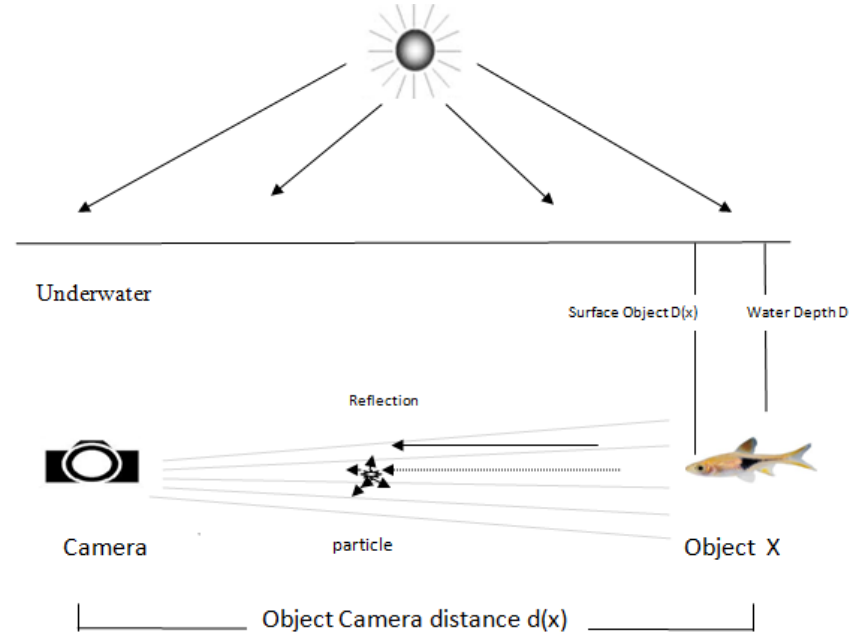

Fig 1. Light Scattering Natural light enters from air to an underwater scene point $X$. The light reflected propagates distance $\mathrm{d}(\mathrm{x})$ to the camera interacts with particles causing reflection and deflection.

\section{Light and Color Loss}

Water is approximately 800 times denser than air, and this density absorbs light - quickly. Not only does this result in dull, monotone colors, but it also reduces contrast and image sharpness. New underwater photographers often get disturbed from the blue / gray hue of their images - a direct result of the properties of water and the affect of light absorption. This is really a very common problem and there are several approaches to reintroducing color and clarity to your images. 


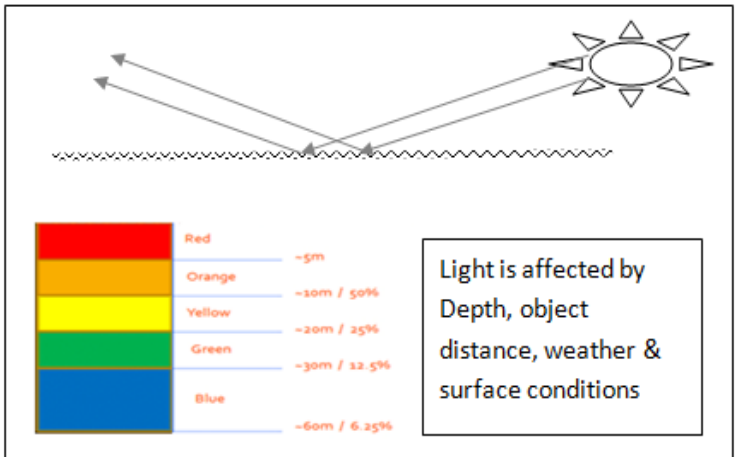

Fig.2. Light Loss

Exact frequencies of ambient light get absorbed at diverse depths, from the longest wavelength to shortest. Red almost disappears at about 5 meters, trailed by orange at 10 meters, yellow at about 20 meters, green at 30 meters and eventually even blue at about 60 meters.

Color Loss Water particles interact with light by absorbing certain wave lengths. Firstly the reds and oranges disappear, later the colour yellows, greens and purples and last the blue. Loss ofcolour red is dramatic and is already noticeable at $50 \mathrm{~cm}$. At 5 metres depth some $90 \%$ has got disappeared. Since the loss of colour varies critically with distance, it is required to make corrections by applying colour correction filters. Their use is explained later on and their effect is quite substantial..The picture shows that it is the total light path that matter.

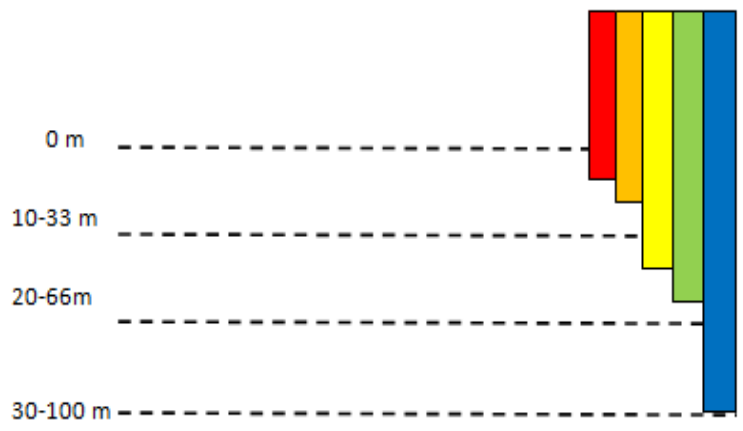

Fig 3. Loss of Color with respect to depth of water

\section{Our Approach}

Our study will be focused on wavelength compensation and dehazing analysis, the auto level adjustment and manual adjustment to enhance the underwater habitat images. The proposed WCID algorithm proceeds in a direction inverse to the underwater image formation path discussed above as depicted in Fig. First, consider the possible presence of the artificial light supply. Next, eliminate the light spreading and color transform that occurred along the course of propagation from the object to the camera. Finally, compensate the inequalities of wavelength attenuation for traversing the water depth to the top of the image and fine-tune the energy loss by deriving a more precise depth value for every point within an image. The common approach for estimating the depth of objects within a scene, i.e., depth map, often requires two images for parallax .In a hazy environment, haze increases with distance; therefore, haze itself can be a useful depth clue for scene understanding. Consequently, evaluating the concentration of haze in a single image is sufficient to predict the distance between the object in the scene and the camera .The darkchannel prior, which is an existing scene-depth derivation method,is used to calculate the depth. 


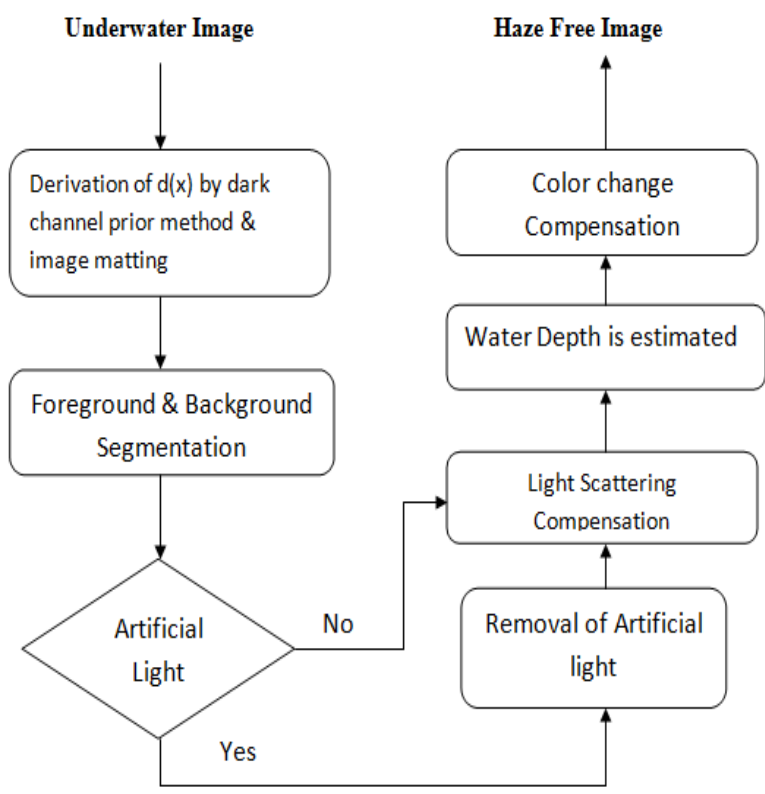

FIG 4. FLOWCHART OF WCID ALGORITHM

\section{EXPERIMENTS AND RESULTS}

The actual input image applied is blur and thus some of the information is being missed. The algorithm based on wavelength compensation and image dehazing which we have introduced does not need any information of the image, and does not rely on the image size. It is clear from the input image Fig. 1. that the image is full of haze and it has lost its brightness i.e it has lost its original color. Hazing and bluish effects is caused by light scattering and color change in underwater images.

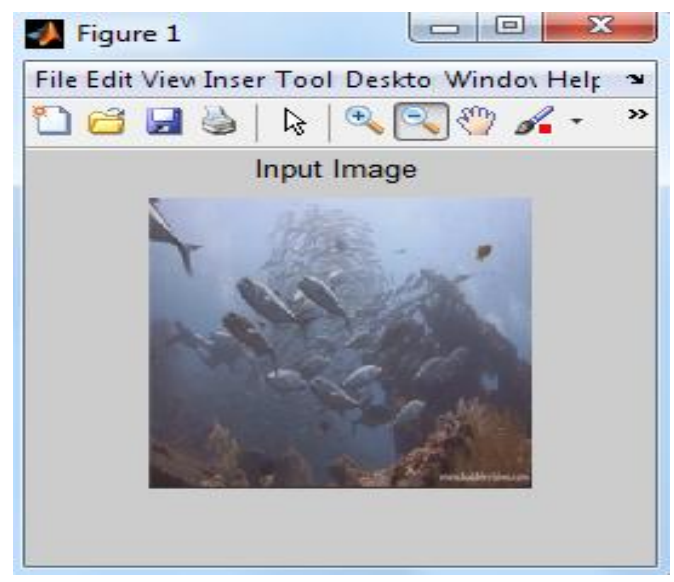

Fig.5. Actual Input Image

Haze increases with distance, thus haze itself can be a useful depth clue for scene understanding. The front and the back scene in an image are segmented as shown in Fig. 6. Consequently, evaluating the deliberation of haze in a single image is adequate to guess the distance between the view and the camera .The background light increases as the object is placed farther away from the camera. Once the scene depth, i.e., object camera distance , is known through the dark-channel prior method, the value of residual energy ratio after wavelength attenuation can be calculated. 


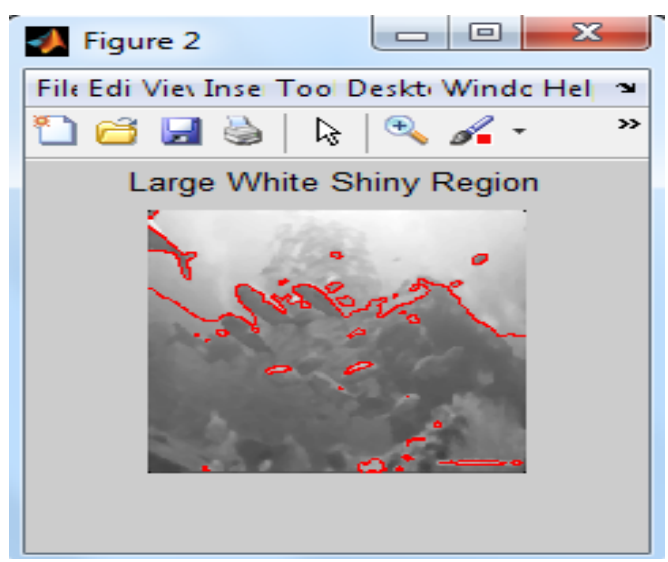

Fig.6. Scene Segmentation

The distance between object and camera is known and image segmentation is done i.e. depth map is calculated using dark Channel prior an existing scene depth method is used to calculate the depth map. Block based darkchannel prior will produce a less accurate depth map. By applying image matting along with the depth map as shown accuracy could be obtained and object contours can be identified more precisely. Applying image matting to the underwater depth map derived by the general dark-channel methodology is a novel approach.

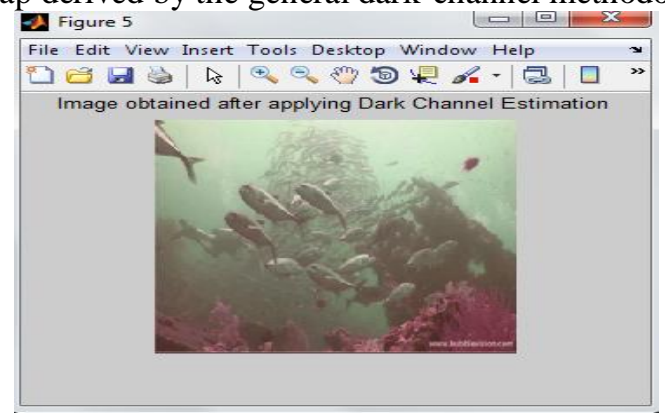

Fig.7.a. Image obtained by Dark Channel Estimation method

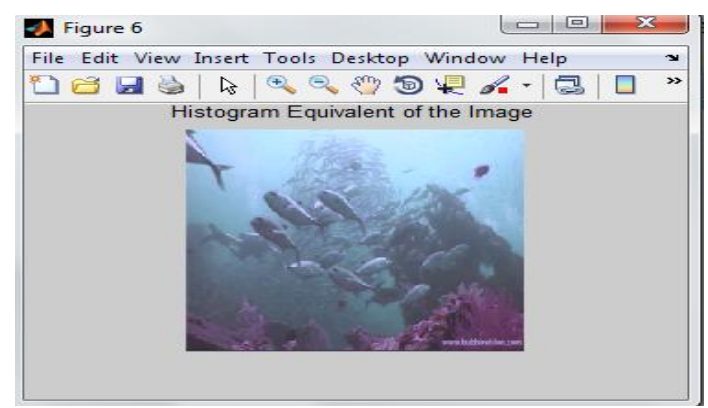

Fig. 7.b. Image obtained by Histogram Equivalent method

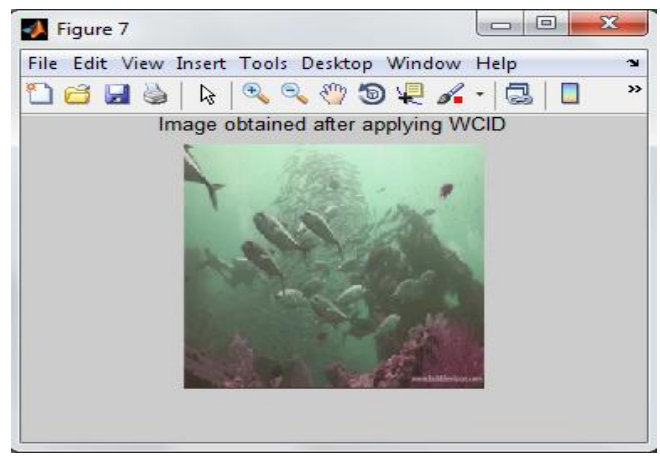

Fig.7.c. Image Obtained by WCID method 
Fig.7. (a) Image obtained after processing with WCID method (b) Image obtained after processing with Dark-channel-based dehazing algorithm and (c) Image obtained after processing with Histogram Equalization method.

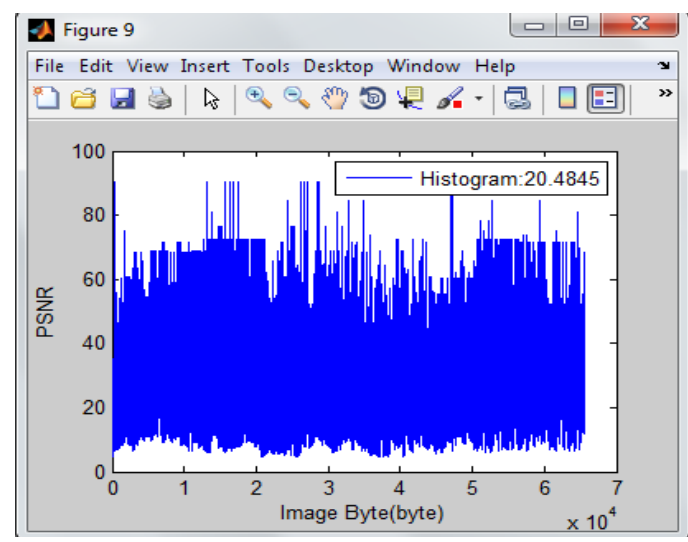

Fig.8.a. PSNR value by Histogram Equivalent Method

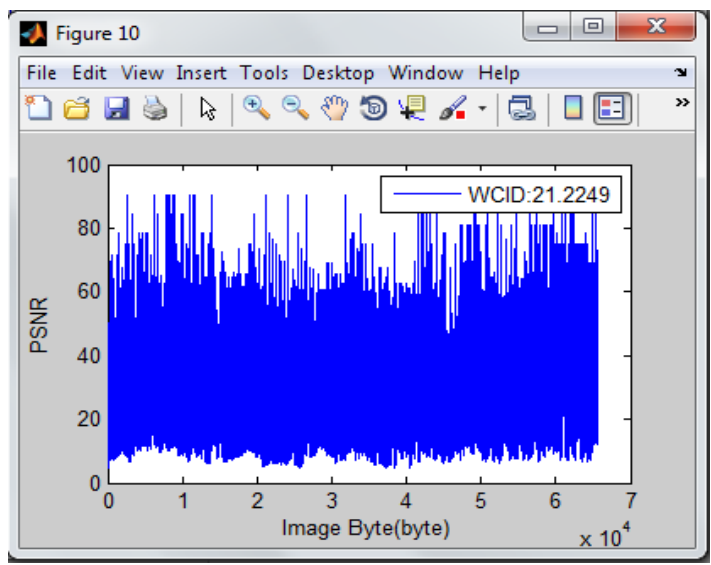

Fig. 8.b PSNR value by WCID Method

Fig 8. (a) PSNR values obtained after processing with Histogram Equalization method (b) PSNR values obtained after processing with WCID Method

GROUND-TRUTH PSNR values of WCID method and Histogram Equilization method are listed in Table 1

\begin{tabular}{|c|c|}
\hline $\begin{array}{c}\text { WCID } \\
\text { METHOD }\end{array}$ & $\begin{array}{c}\text { HISTOGRAM } \\
\text { EQUILIZATION METHOD }\end{array}$ \\
\hline 21.2249 & 20.4845 \\
\hline
\end{tabular}

Table 1

Other than taking the artificial light source, the object- camera distance, and the water depth into consideration, Fig. 7.a, Fig. 7.b and Fig. 7.c shows different methods to enhance the underwater image. It could be clearly seen from the output that the output generated by WCID method is far better as compared to Dark Channel Estimation and Histogram Equivalent method. The image obtained with Histogram method is somewhat gray in color whereas with dark Channel Estimation Method has lost its color. In addition the peak signal noise ratio PSNR value for the given input image by the Histogram Equivalent Method Fig. 8.a is found to be 20.4845 where the PSNR values by WCID method Fig.8.b is found to be 21.2249. Thus it is clear from the above output that WCID method is better than the above approaches. As shown in Table I, the WCID proposed obtains the highest PSNR value in both depths. In addition, the performance of WCID is the most robust through different water depths due to the incorporation of wavelength compensation, image dehazing, and artificial lighting removal. On the other hand, the PSNR value obtained by histogram equalization drops significantly as the depth 
increases. This is due to the fact that the dynamic range of the red color component decreases substantially with the depth due to wavelength attenuation. An attempt, without taking dehazing or wavelength compensation into consideration, blindly expanding the range of color components will adversely accompany with a huge amount of error. The relatively low value of the PSNR can be attributed to the presence of water bubbles, the misalignment in matching color patches with different dimensions and orientations, and water permeated through the seam between the green color patch and the board, making it distinct from the counterpart in the ground-truth image.

\section{CONCLUSION}

The WCID algorithm proposed in this paper can effectively restore image color balance and remove haze. To the best of our knowledge, no existing techniques can handle light scattering and color change along with the artificial light removing distortions suffered by underwater images simultaneously. The experimental results demonstrate superior haze removing and color balancing capabilities of the proposed WCID over traditional dehazing and histogram equalization methods. We propose an original idea to reconstruct a submerged object distorted by moving water surface. The Wavelength compensation and dehazing technique is employed to recover the phase of the true object. Although experiments demonstrate that our approach is promising, there exist some limits. One boundary is that our algorithm needs a large computer memory and heavy computation. Another limit is the recursive phase recovery method with only a subset of the phase information. This may lessen the resolution of the output. To overcome such limits is the next step in our research exist some limits. One boundary is that our algorithm needs a large computer memory and heavy computation. Another limit is the recursive phase recovery method with only a subset of the phase information. This may lessen the resolution of the output. To overcome such limits is the next step in our research.

\section{REFERENCES}

[1] John Y. Chiang and Ying-Ching Chen, "Underwater Image Enhancement by Wavelength Compensation and Dehazing" IEEE Transactions on Image Processing, Vol. 21, No. 4, April 2012

[2] K. Lebart, C. Smith, E. Trucco, and D. M. Lane, "Automatic Indexing of Underwater Survey Video: Algorithm and Benchmarking Method,” IEEE J. Ocean. Eng., Vol. 28, No. 4, Pp. 673-686, Oct. 2003.

[3] Baharum B Baharudin, "Significance Level of Image Enhancement Techniques for Underwater Images" 2012 International Conference on Computer \& Information Science (Iccis)978-1-4673-1938-6112/\$31.00 @20 12 IEEE

[4] Kaiming He, Jian Sun,Xiaoou Tang, "Single Image Haze Removal using Dark Channel Prior" Vol.3 Issue.2, February- 2014, Pg. 603-605

[5] Rajesh Garg, Bhawna Mittal, Sheetal Garg, "Histogram Equalization Techniques for Image Enhancement"

[6] Http://Science.Kennesaw.Edu/ Jdirnber/Limno/Lecphy/Lecphy.Html

[7] Yuzhang Chen, Min Xia, Wei Li, Xiaohui Zhang, Kecheng Yang, "Comparison of Point Spread Models for Underwater Image Restoration" Journal Homepage: www.Elsevier.De/Ijleo

[8] C. J. Prabhakar and P. U. Praveen Kumar, "Image Enhancement and Restoration Methods for Underwater Images"

[9] Omar Oreife, Guangshu, Teresa Pace, And Mubarak Shah, "A Two-Stage Reconstruction Approach for Seeing Through Water", Computer Vision and Pattern Recognition (Cvpr), 2011 IEEEconference

[10] Yulin Zhang, Xia Zhu, "Image De-Noising Algorithm Study and Realization Based on Wavelet Analysis" ,978-1-4244-69437/10/\$26.00 @2010 IEEE

[11] Yoav Y. Schechner And Nir Karpel, “Recovery of Underwater Visibility and Structure by Polarization Analysis”, IEEE Journal of Oceanic Engineering”, Vol. 30, No. 3, July 2005

[12] Fang Wang, Tianxu Zhang, Jinpeng Deng, "A Novel Method Concerning about Image Transition Region Extraction And Segmentation",978-1-61284-774-0/11/\$26.00 @2011 IEEE

[13] Anthoni J Floor, "Water and Light in Underwater Photography" Http://www.seafriends.org.nz/phgraph/water.htm (2005)

[14] Joseph Tepper Divev Photo Guide http://www.divephotoguide.com/getting-started-with underwater-photography/basic-principleslight-underwater/

[15] Pooja Sahu, Neelesh Gupta, Neetu Sharma, “A Survey on Underwater Image Enhancement Techniques”, International Journal of Computer Applications (0975 - 8887) Volume 87 - No.13, February 2014

[16] Daisuke Miyazaki, Daisuke Akiyama, Masashi Baba, Ryo Furukawa, Shinsaku Hiura, Naoki Asada, "Polarization-based dehazing using two reference object, ICCV2013 http://ime.info.hiroshima-cu.ac.jp/

[17] Carlo Dal Mutto, Pietro Zanuttigh, Guido M. Cortelazzo, "Scene Segmentation by Color and Depth Information and its Applications", International Journal of Computer Vision, 47:7-42, 2001.

[18] Dinesh Sonker, M. P. Parsa, "Comparison of Histogram Equalization Techniques for Image Enhancement of Grayscale images of Dawn and Dusk”, (IJMER) www.ijmer.com Vol. 3, Issue. 4, Jul - Aug. 2013 pp-2476-2480 ISSN: 2249-6645 